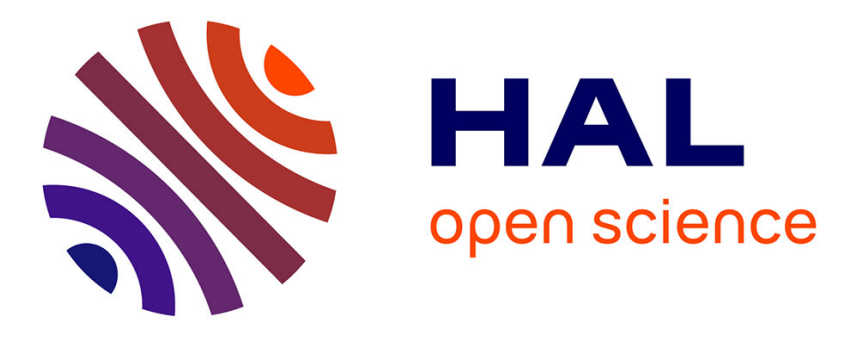

\title{
Limits of variance-based sensitivity analysis for non- identifiability testing in high dimensional dynamic models
}

Simona Dobre, Thierry Bastogne, Christophe Profeta, Muriel Barberi-Heyob, Alain Richard

\section{To cite this version:}

Simona Dobre, Thierry Bastogne, Christophe Profeta, Muriel Barberi-Heyob, Alain Richard. Limits of variance-based sensitivity analysis for non- identifiability testing in high dimensional dynamic models. Automatica, 2012, 48 (1), pp.2740-2749. 10.1016/j.automatica.2012.05.004 . hal-00730316

\section{HAL Id: hal-00730316 https://hal.science/hal-00730316}

Submitted on 10 Sep 2012

HAL is a multi-disciplinary open access archive for the deposit and dissemination of scientific research documents, whether they are published or not. The documents may come from teaching and research institutions in France or abroad, or from public or private research centers.
L'archive ouverte pluridisciplinaire HAL, est destinée au dépôt et à la diffusion de documents scientifiques de niveau recherche, publiés ou non, émanant des établissements d'enseignement et de recherche français ou étrangers, des laboratoires publics ou privés. 


\title{
Limits of variance-based sensitivity analysis for non- identifiability testing in high dimensional dynamic models
}

\author{
Simona Dobre ${ }^{\mathrm{a}}$, Thierry Bastogne ${ }^{\mathrm{a}, \mathrm{b}}$, Christophe Profeta ${ }^{\mathrm{c}}$, \\ Muriel Barberi-Heyob ${ }^{\mathrm{d}}$, Alain Richard ${ }^{\mathrm{a}}$ \\ ${ }^{a}$ Centre de recherche en Automatique de Nancy (CRAN), Nancy-Université, CNRS UMR 7039, Campus Science, BP 70239, \\ 54506, Vandouvre-lès-Nancy Cedex, France \\ b BIGS (INRIA Nancy Grand-Est) \\ ${ }^{\mathrm{c}}$ Institut Elie Cartan, Nancy-Université,Campus Science, BP 70239, 54506, Vandœuvre-lès-Nancy Cedex, France \\ ${ }^{\mathrm{d}}$ Centre de recherche en Automatique de Nancy (CRAN), Nancy-Université, CNRS UMR 7039, Centre Alexis Vautrin, \\ 54511, Vandouvre-lès-Nancy Cedex, France
}

\begin{abstract}
In systems biology, a common approach to model biological processes is to use large systems of nonlinear differential equations. The associated parameter estimation problem then requires a prior handling of the global identifiability question in a realistic experimental framework. The lack of a method able to solve this issue has indirectly encouraged the use of global sensitivity analysis to select the subset of parameters to estimate. Nevertheless, the links between these two global analyses are not yet fully explored.
\end{abstract}

The present work reveals new bridges between sensitivity analyses and global non-identifiability, through the use of functions derived from the Sobol' high dimensional representation of the model output. We particularly specify limits of variance-based sensitivity tools to completely conclude on global non-identifiability of parameters in a given experimental context.

Key words: identifiability, sensitivity analysis, nonlinear systems, dynamical systems, systems biology.

\section{Introduction}

In systems biology, the inference of biological networks from quantitative properties of their elementary constituents is a major area of research $[4,9]$. This raises particular challenges such as the identification of highdimensional nonlinear dynamical systems and more precisely the analysis of their parameter identifiability $[13,19,27]$.

Different classifications of parameter identifiability definitions exist. We refer herein to three classes (see Fig. 1): a priori identifiability, a posteriori identifiability and practical identifiability. The first class, known also as the theoretical or structural identifiability of model parameters, examines the question of existence and uniqueness of a solution to the parameter estimation problem [30], in an idealized framework where (1) the system and model have identical structure (no characterization error); (2) the data are noise-free, and (3) the input signals and measurement times can be chosen at will. However, this is only a necessary condition which can not guarantee successfull parameter estimation from real data. The second class, namely the a posteriori identifiability only considers the first two working assumptions and is a particular case of the output distinguishability [6] for a finite collection of noise-free observations and a given input signal. The last class, practical identifiability, only relies on the first hypothesis and accounts for the noise factor but is generally established for a given estimation criterion $[5,29]$. For that reason, this class of identifiability is often linked to the theory of optimization in mathematics.

Sensitivity analysis of the model output with respect to changes in model parameters is another technique widely used in system modeling to discriminate influential and non influential parameters $[22,28]$. Dynamic sensitivity analysis has already been applied to biological networks for various purposes such as experimental design [23], 
Table 1

Nomenclature

\begin{tabular}{|c|c|}
\hline Symbol & Definition \\
\hline$t_{k}$ & the $\mathrm{k}^{\text {th }}$ time instant, with $k=0, \ldots, N-1$ \\
\hline $\mathbf{p} \in \mathbb{P} \subset \mathbb{R}^{n}$ & the parameters of the model \\
\hline $\mathbf{p}_{\sim i}$ & $\begin{array}{l}\text { the vector composed by all the parameters } \\
\text { except } p_{i}\end{array}$ \\
\hline $\mathbf{u} \in \mathbb{R}^{n_{u}}$ & input signals \\
\hline $\mathbf{x} \in \mathbb{R}^{n_{x}}$ & state variables \\
\hline $\mathbf{y} \in \mathbb{R}^{n_{y}}$ & output variables \\
\hline $\mathrm{E}[\cdot]$ & the expectation operator \\
\hline $\mathrm{V}[\cdot]$ & the variance operator \\
\hline$V_{i_{1}, \ldots, i_{r}}\left(t_{k}\right)$ & $\begin{array}{l}\text { the } \mathrm{r}^{\text {th }} \text {-order variance of the output } y \text { with } \\
\text { respect to collective effect of the parame- } \\
\text { ters } p_{i_{1}}, \ldots, p_{i_{r}}\end{array}$ \\
\hline$S_{i_{1}, \ldots, i_{r}}\left(t_{k}\right)$ & $\begin{array}{l}\text { the } \mathrm{r}^{\mathrm{th}} \text {-order sensitivity of the output } y \\
\text { with respect to collective effect of the pa- } \\
\text { rameters } p_{i_{1}}, \ldots, p_{i_{r}}\end{array}$ \\
\hline $\mathbf{I}^{n}=[0,1]^{n}$ & $n$-dimensional unit hypercube \\
\hline$\Psi_{i}\left(t_{k}, \mathbf{p}\right)$ & $\begin{array}{l}\text { the total effect on the model output } y \text { of } \\
\text { the parameter } p_{i}\end{array}$ \\
\hline$\Omega_{i}\left(t_{k}, \mathbf{p}_{\sim j}\right)$ & $\begin{array}{l}\text { represents the influence on the output } y \text { of } \\
\text { the parameter } p_{i} \text { independently of } p_{j}\end{array}$ \\
\hline
\end{tabular}

parameter estimation [12] or the analysis of oscillatory systems $[18,34]$.

Several investigations on the connections between dynamic sensitivity and parameter identifiability analyses have been carried out $[2,27,32]$, but the latter were only focused on local analysis.

In genomics, proteomics or metabolomics, biological parameters may vary widely within different ranges. As a consequence, global a posteriori identifiability needs to be addressed. Unfortunately, there is no technique able to assess the global identifiability condition in a given experimental context, i.e. when the input signal and the sampling conditions are imposed by the experimental context. Consequently, authors generally prefer to apply global sensitivity analysis techniques without solid justifications related to identifiability. Indeed, while the relationship between local sensitivity and identifiability analysis, through the Fisher information matrix, is clearly established $[3,31,33]$, the link between global studies is less obvious. As a matter of fact, only insensitive parameters are generally considered as being nonidentifiable. This is not surprising since global sensitivity measures usually serve as model reduction principles (before parameter estimation) or in tandem with uncertainty analysis for model robustness analysis [22]. However, sensitive parameters could also be non-identifiable.

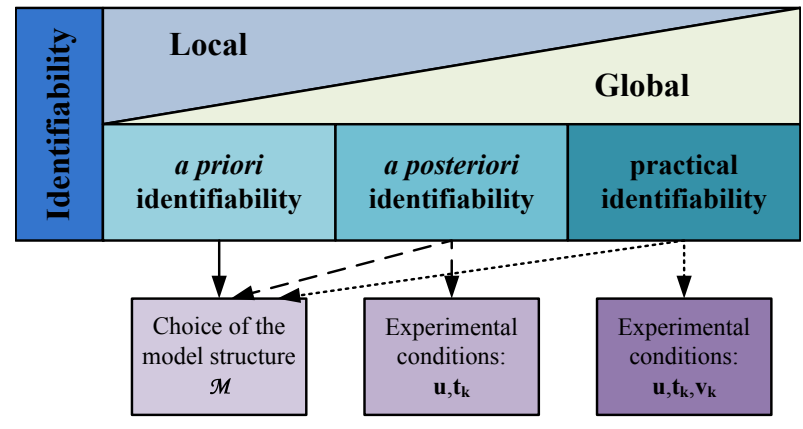

Fig. 1. Classification of identifiability definitions, where $\mathcal{M}$ denotes the model structure, $\mathbf{u}$ the input signals, $t_{k}$ the time measurements and $v_{k}$ the output noise

The objective of this paper is to present new results on the connections between global a posteriori identifiability and global dynamic sensitivity analysis. This study is structured around the Sobol' decomposition ${ }^{1}$ method [24]. Specific functions, entitled $\Psi$ and $\Omega$-functions, derived from the Sobol' high dimensional model respresentation, are introduced. Their linear time-dependence and injectivity are examined, and their consequences on the non-identifiability of parameters are discussed. We show that variance-based sensitivity analysis can be used to test only one out of three causes of nonidentifiability in a given experimental context, where the input signal and the mesurement times are imposed. We also point out that the conclusions on parameter nonidentifiability, in the case of colinear sensitivity measures, must be treated with caution.

This paper is structured as follows: a priori and a posteriori identifiabilities are firstly defined. The global sensitivity analysis based on the Sobol' high dimensional model representation is then briefly introduced in Section 3. Finally, the main contributions of this study are presented in Section 4 in which the links between sensitivity and global identifiability analyses are decomposed, in a theoretical framework.

\section{Identifiability analysis}

Let us consider a dynamic system described by a nonlinear state-space model defined as follows:

$$
\left\{\begin{array}{l}
\frac{d}{d t} \mathbf{x}(t)=\mathrm{f}(\mathbf{x}(t), \mathbf{u}(t), t, \mathbf{p}) ; \mathbf{x}(0)=\mathbf{x}_{0}(\mathbf{p}) \\
\mathbf{y}(t, \mathbf{p})=\mathrm{h}(\mathbf{x}(t), \mathbf{p})
\end{array}\right.
$$

where $\mathbf{x} \in \mathbb{R}^{n_{x}}, \mathbf{u} \in \mathbb{R}^{n_{u}}$ and $\mathbf{y} \in \mathbb{R}^{n_{y}}$ denote the state, input and output vectors respectively. The variable $\mathbf{x}_{0}$ is the initial value of the state vector, $\mathbf{p} \in \mathbb{R}^{n}$ is the vector

\footnotetext{
1 Decomposition known as the Hoeffding decomposition [7], HDMR (high dimensional model representation) expansion [17] or more recently, as the Sobol' decomposition [26].
} 
of model parameters and $t$ is the time variable. $\mathrm{f}(\cdot)$ and $\mathrm{h}(\cdot)$ contain the state and output equations respectively.

In a priori identifiability, the solution uniqueness of the parameter estimation problem is assessed in an idealized framework [30]. However, in experimental biology, the input design is often subject to economical and/or technical constraints and the number of observations is frequently limited to a few data points collected at time instants $\left\{t_{k}\right\} \in \mathbb{T}, k=0, \ldots, N-1$. In such restrictive experimental frameworks, even if a parameter is a priori identifiable, it may not be so in practice, due to a lack of information in the available observations. The a posteriori identifiability condition can be stated as follows: given a parametric model structure with given input signals $\mathbf{u}$ and initial conditions $\mathbf{x}_{0}$, a parameter $p_{i}$, with $i \in 1, \ldots, n$ is a posteriori identifiable, if for almost all $\mathbf{p}^{*} \in \mathbb{P} \subset \mathbb{R}^{n}$ with $n$ the number of model parameters, the following condition is satisfied

$$
\mathbf{y}\left(t_{k}, \mathbf{p}\right)=\mathbf{y}\left(t_{k}, \mathbf{p}^{*}\right) \forall t_{k} \in \mathbb{T} \Longrightarrow p_{i}=p_{i}^{*}
$$

The local a posteriori identifiability, corresponding to $\mathbf{p}^{*} \in \mathbb{V}(\mathbf{p})$ where $\mathbb{V}(\mathbf{p})$ denotes the neighborhood of $\mathbf{p}$, is not considered here since it is not relevant to biological models, for which parameters may vary over wide ranges. Several methods exist to analyze global a priori identifiability, based on state isomorphisms [15], differential algebra $[1,11,20,21]$ or power series expansions $[16,30]$. Unfortunately, there is no available approach to assess $a$ posteriori global identifiability. This lack of a practical solution has encouraged researchers in systems biology to use techniques of global sensitivity analysis $[10,14]$.

\section{Global sensitivity analysis}

Several categories of sensitivity analysis methods already exist in previously published studies [22]. Herein, we only focus on variance-based global methods and more precisely on the Sobol' sensitivity method [24]. This method allows the computation of the output's sensitivity with respect to the variation of model parameters over the entire parametric domain. The sensitivity measure related to a certain parameter is evaluated while varying all other parameters as well, revealing thus any existing interaction. Hereafter, we apply this method to dynamic systems, adjusting the terminology from sensitivity indices for static systems to sensitivity functions for dynamic ones.

\section{Hypotheses of the method:}

$\left(\mathcal{H}_{1}\right)$ the $n$ parameters are considered as i.i.d. random variables uniformly distributed over the ndimensional unit cube $\mathbf{I}^{n}=[0,1]^{n}$, i.e., $p_{i} \sim$ $\mathcal{U}[0,1], \forall i \in\{1, \ldots, n\} ;$

$\left(\mathcal{H}_{2}\right) y(t, \mathbf{p})$ is continuously differentiable and square integrable;
$\left(\mathcal{H}_{3}\right)$ when analyzing the global sensitivity analysis w.r.t. parameters, all other computational factors which could affect the model output, such as the simulation method, the sampling time, the input signal, etc., are not modified during the analysis.

As global sensitivity analysis with respect to parameters is generally computed independently for each model output, for the sake of readability we will consider hereafter that we are dealing with a single output model. Furthermore, knowing that we are working with dynamical systems and that we are interested in expressing the variance and sensitivity functions for specific time measurements, all the equations will be written for a specific time instant $t_{k} \in \mathbb{T}$ (but are, of course, valid for every $\left.t_{k} \in \mathbb{T}\right)$.

The main idea behind Sobol' decomposition is the expansion of the model output variable $y\left(t_{k}, \mathbf{p}\right)$ into summands of increasing dimensionality, namely

$$
\begin{aligned}
y\left(t_{k}, \mathbf{p}\right)= & y_{0}\left(t_{k}\right)+\sum_{i=1}^{n} y_{i}\left(t_{k}, p_{i}\right)+\sum_{i=1}^{n-1} \sum_{j>i}^{n} y_{i, j}\left(t_{k}, p_{i}, p_{j}\right) \\
& +\ldots+y_{1, \ldots, n}\left(t_{k}, p_{1}, \ldots, p_{n}\right)
\end{aligned}
$$

$\forall t_{k} \in \mathbb{T}$. For (3) to hold, this decomposition has the following properties:

$\left(\mathcal{P}_{1}\right)$ the term $y_{0}\left(t_{k}\right)$ must be constant w.r.t. model parameters $\mathbf{p}$, i.e.

$$
y_{0}\left(t_{k}\right)=\int_{\mathbf{I}^{n}} y\left(t_{k}, \mathbf{p}\right) \mathrm{d} \mathbf{p} \stackrel{\left(\mathcal{H}_{1}\right)}{=} \mathrm{E}[y]
$$

with $\mathrm{E}[\cdot]$ being the expectation operator.

$\left(\mathcal{P}_{2}\right)$ the integrals of every summand over any of its dependent parameters must be zero, i.e.

$$
\begin{aligned}
& \int_{0}^{1} y_{i_{1}, \ldots, i_{r}}\left(t_{k}, p_{i_{1}}, \ldots, p_{i_{r}}\right) d p_{l}=0 \stackrel{\left(\mathcal{H}_{1}\right)}{=} \mathrm{E}\left[y_{i_{1}, \ldots, i_{r}}\right] \\
& \text { with } l \in\left\{i_{1}, \ldots, i_{r}\right\}, 1 \leq i_{1}<\ldots<i_{r} \leq n
\end{aligned}
$$

A consequence of these two properties is the orthogonality of all the terms in (3), i.e. if $\left\{i_{1}, \ldots, i_{r}\right\} \neq\left\{j_{1}, \ldots, j_{s}\right\}$ and $1 \leq j_{1}<\ldots<j_{s} \leq n[24]$, then

$$
\int_{\mathbf{I}^{n}} y_{i_{1}, \ldots, i_{r}}\left(t_{k}, p_{i_{1}}, \ldots, p_{i_{r}}\right) \cdot y_{j_{1}, \ldots, j_{s}}\left(t_{k}, p_{j_{1}}, \ldots, p_{j_{s}}\right) d \mathbf{p}
$$

is also null. Moreover, if $y\left(t_{k}, \mathbf{p}\right)$ is square integrable (hypothesis $\left.\mathcal{H}_{2}\right)$, then its variance, denoted $V\left(t_{k}\right)$, is given 
by

$$
\begin{aligned}
V\left(t_{k}\right)= & \int_{\mathbf{I}^{n}}\left(y^{2}\left(t_{k}, \mathbf{p}\right)-y_{0}^{2}\left(t_{k}\right)\right) d \mathbf{p} \\
= & \int_{\mathbf{I}^{n}}\left(\sum_{i=1}^{n} y_{i}^{2}\left(t_{k}, p_{i}\right)+\sum_{i=1}^{n-1} \sum_{j>i}^{n} y_{i, j}^{2}\left(t_{k}, p_{i}, p_{j}\right)+\right. \\
& \left.+\ldots+y_{1, \ldots, n}^{2}\left(t_{k}, p_{1}, \ldots, p_{n}\right)\right) d \mathbf{p}
\end{aligned}
$$

Based on the hypothesis $\left(\mathcal{H}_{1}\right)$ and the property $\left(\mathcal{P}_{1}\right)$, we can further write:

$$
V\left(t_{k}\right)=\sum_{i=1}^{n} V_{i}\left(t_{k}\right)+\sum_{i=1}^{n-1} \sum_{j>i}^{n} V_{i, j}\left(t_{k}\right)+\ldots+V_{1, \ldots, n}\left(t_{k}\right) .
$$

This equation is also known as the ANOVA-HDMR decomposition, where

$V_{i}$ represents the variance of the output due to the direct effect of the parameter $p_{i}$, defined as

$$
V_{i}\left(t_{k}\right)=\mathrm{V}\left[y_{i}\left(t_{k}, p_{i}\right)\right]=\mathrm{V}_{p_{i}}\left[\mathrm{E}\left[y \mid p_{i}=p_{i}^{*}\right]\right]
$$

with $\mathrm{E}\left[y \mid p_{i}=p_{i}^{*}\right]$ the conditional expectation of $y$ over all the parameters but $p_{i}$ which is fixed to a particular value $p_{i}^{*}$ and $\mathrm{V}_{p_{i}}[\cdot]$ the variance over the distribution of $p_{i}$.

$V_{i, j}$ represents the joint effect of the parameters $p_{i}$ and $p_{j}$ on the output $y$ and is defined as

$$
V_{i, j}\left(t_{k}\right)=\mathrm{V}\left[y_{i, j}\left(t_{k}, p_{i}, p_{j}\right)\right]
$$

Analogous equations can be written for the other high-order terms in (4).

Dividing both sides of the ANOVA-HDMR decomposition in (4) by $V\left(t_{k}\right)$ gives

$$
1=\sum_{i=1}^{n} S_{i}\left(t_{k}\right)+\sum_{i=1}^{n-1} \sum_{j>i}^{n} S_{i, j}\left(t_{k}\right)+\ldots+S_{1, \ldots, n}\left(t_{k}\right)
$$

where

- $S_{i}\left(t_{k}\right)$ is the $\mathrm{i}^{\text {th }}$ first-order sensitivity function (known also as the marginal sensitivity function), defined by

$$
S_{i}\left(t_{k}\right)=V_{i}\left(t_{k}\right) / V\left(t_{k}\right),
$$

and representing the single effect of the parameter $p_{i}$ on the variance of the output;

- and $S_{i_{1}, \ldots, i_{r}}\left(t_{k}\right)$ is the $\mathrm{r}^{\text {th }}$ order sensitivity function,

$$
S_{i_{1}, \ldots, i_{r}}\left(t_{k}\right)=V_{i_{1}, \ldots, i_{r}}\left(t_{k}\right) / V\left(t_{k}\right)
$$

representing the sensibility w.r.t. a group of parameters in interaction (also called the collective sensitivity function).
Homma and Saltelli have introduced in [8] the definition of total sensitivity functions, regrouping the sensitivity of the model output with respect to the influence of a parameter in the different forms (direct and interactions with other parameters). These functions are defined as

$$
S_{T_{i}}\left(t_{k}\right)=V_{T_{i}}\left(t_{k}\right) / V\left(t_{k}\right)
$$

where $V_{T_{i}}\left(t_{k}\right)$ is the total variance in relation to the influence of parameter $p_{i}$, i.e

$$
V_{T_{i}}\left(t_{k}\right)=V_{i}\left(t_{k}\right)+\sum_{j=1}^{n} V_{i, j}\left(t_{k}\right)+\ldots+V_{1, \ldots, n}\left(t_{k}\right) .
$$

\section{Implications of global sensitivity and identifi- ability analyses}

There is little reference in literature for the connections between global sensitivity analysis results (more precisely, variance-based analysis results) and parameter (non-) identifiability. Mainly, they refer to a general acknowledged link : null total sensitivity also implies the non-identifiability of the parameter in question. Nevertheless, a 'sensitive' parameter could also be nonidentifiable in practice.

This section gathers the principal theoretical results. First of all, we will introduce new functions denoted by $\Psi$, derived from the Sobol' decomposition and employed to characterize the complete action on the model output of a parameter $p_{i}$. Then, studying the properties of the $\Psi$ functions, we will illustrate three causes that lead to the lack of identifiability:

(a) null total sensitivity function, $S_{T_{i}}$

(b) non-injectivity of the $\Psi_{i}$ functions w.r.t. parameter $p_{i}$

(c) colinear sensitivity functions.

In order to illustrate this point, several academic examples will be introduced.

\subsection{Definition of $\Psi$ functions}

Let us consider the $\Psi_{i}$ function representing the total effect on the model output of the parameter $p_{i}$. In other terms, this function gathers all the Sobol' decomposition terms in eq. (3) involving the index $i$ :

$$
\begin{aligned}
\Psi_{i}\left(t_{k}, \mathbf{p}\right)= & y_{i}\left(t_{k}, p_{i}\right)+\sum_{j \neq i}^{n} y_{i, j}\left(t_{k}, p_{i}, p_{j}\right)+\ldots+ \\
& +y_{1, \ldots, i, \ldots, n}\left(t_{k}, p_{1}, \ldots, p_{i}, \ldots, p_{n}\right) .
\end{aligned}
$$


These functions provide the means of analyzing properties like insensitivity, non-injectivity of the model output w.r.t. the parameter $p_{i}$, and the compensation of the actions of two parameters $p_{i}$ and $p_{j}$.

Taking into account the properties $\left(\mathcal{P}_{1}\right)$ and $\left(\mathcal{P}_{2}\right)$ of the Sobol' expansion (3), we have the following property, for all $t_{k} \in \mathbb{T}$,

$$
\int_{0}^{1} \Psi_{i}\left(t_{k}, \mathbf{p}\right) d p_{i}=0 .
$$

Furthermore, the total variance function w.r.t. the parameter $p_{i}$, can be also expressed as

$$
V_{T_{i}}\left(t_{k}\right)=\int_{\mathbf{I}^{n}} \Psi_{i}^{2}\left(t_{k}, \mathbf{p}\right) d \mathbf{p}
$$

\subsection{Case (a): null total sensitivity functions}

Even if the link between null total sensitivity functions and parameter non-identifiability is generally acknowledged, we formally address it herein with two propositions.

Proposition 4.1 A total sensitivity function, $S_{T_{i}}\left(t_{k}\right)$, is null iff the function $\Psi_{i}\left(t_{k}, \mathbf{p}\right)$ is also null, and that for all time measurements $t_{k} \in \mathbb{T}$.

Proof Variance and sensitivity functions are all positive definite. Therefore, a null total variance $V_{T_{i}}\left(t_{k}\right)$, or total sensitivity function $S_{T_{i}}\left(t_{k}\right)$, for all time measurements, will also imply the nullity of all the terms involving the index $i$ in the Sobol' decomposition (in eq. (3))[25], i.e.

$$
y_{i}\left(t_{k}, p_{i}\right)=y_{i, j}\left(t_{k}, p_{i}, p_{j}\right)=\ldots=y_{1, \ldots, n}\left(t_{k}, \mathbf{p}\right)=0 .
$$

Considering the special construction of the $\Psi_{i}$ function, defined in eq. (9), we can conclude to the nullity of this function for all the time measurements.

Proposition 4.2 The nullity of the total sensitivity function $S_{T_{i}}$, for all time measurements, implies also the non-identifiability of parameter $p_{i}$.

Proof As seen above, a null total sensitivity function, $S_{T_{i}}\left(t_{k}\right), \forall t_{k} \in \mathbb{T}$, implies the nullity of the $\Psi_{i}$ function. This means that we can express the model output variable as a function of $n-1$ parameters, $\mathbf{p}_{\sim i}$. In other words, $p_{i}$ has no influence on the output and there is at least two distinct parameter values, $\mathbf{p}, \mathbf{p}^{*} \in \mathbb{P}$, such as

$$
y\left(t_{k}, \mathbf{p}\right)=y\left(t_{k}, \mathbf{p}^{*}\right) \forall t_{k} \in \mathbb{T}
$$

with $\mathbf{p}^{*}=\left[p_{1}, \ldots, p_{i}^{*}, \ldots, p_{n}\right]$. The parameter $p_{i}$ is thus non-identifiable.

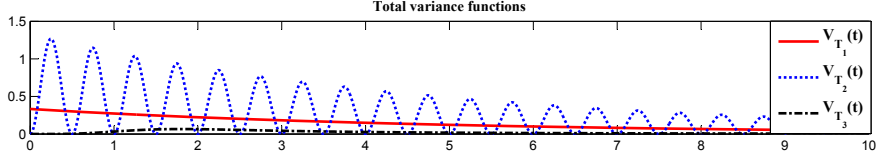

(a)

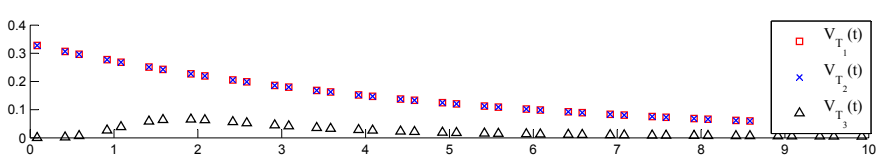

(b)

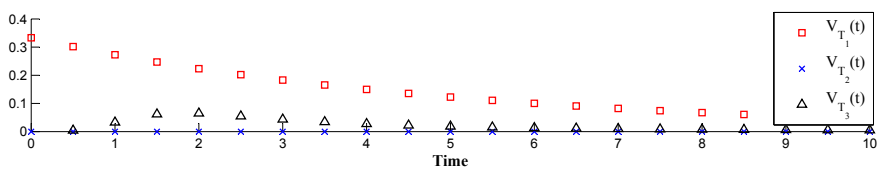

(c)

Fig. 2. Total variance functions of the model expressed in (11), for three different time-sampling strategies: (a) $t_{k}=0.01 \cdot k ;(\mathrm{b}) t_{k}=(1+6 k) / 12$ and (c) $t_{k}=0.5 \cdot k$.

In practice, the parameters with a total sensitivity function inferior (for all time measurements) to an empirical threshold, are generally considered as globally nonidentifiable $[10,22,24]$.

\section{Example 1}

Let us consider a three-parameter model defined as

$$
y\left(t_{k}, \mathbf{p}\right)=\left(2 p_{1}+4 p_{2} \sin \left(2 \pi t_{k}\right)\right) \cdot \mathrm{e}^{-0.1 t_{k}}+\mathrm{e}^{-p_{3} t_{k}^{2}}
$$

with $\mathbf{p} \in \mathbf{I}^{3}$, to illustrate the link between a null (or inferior to an empirical threshold) total sensitivity function and parameter non-identifiability. Hence, the Sobol' decomposition terms are

$$
\begin{aligned}
& y_{1}\left(t_{k}, p_{1}\right)=\left(2 p_{1}-1\right) \cdot \mathrm{e}^{-0.1 t_{k}} \\
& y_{2}\left(t_{k}, p_{2}\right)=2 \cdot\left(2 p_{2}-1\right) \cdot \sin \left(2 \pi t_{k}\right) \cdot \mathrm{e}^{-0.1 t_{k}} \\
& y_{3}\left(t_{k}, p_{3}\right)=\mathrm{e}^{-p_{3} t_{k}^{2}}+\frac{1}{t_{k}^{2}} \cdot\left(\mathrm{e}^{-t_{k}^{2}}-1\right)
\end{aligned}
$$

whereas all the high order summands are, in this case, null.

An a priori identifiability analysis ${ }^{2}$ (through a Taylor series approach) concludes to the global identifiability of the three parameters in a theoretical context. The total

\footnotetext{
2 For space reasons, we did not include herein the details about this identifiability study.
} 
variance functions, obtained by computer algebra, are

$$
\begin{aligned}
& V_{T_{1}}\left(t_{k}\right)=0.33 \cdot \mathrm{e}^{-0.2 t_{k}} \\
& V_{T_{2}}\left(t_{k}\right)=1.33 \cdot \sin \left(2 \pi t_{k}\right)^{2} \cdot \mathrm{e}^{-0.2 t_{k}} \\
& V_{T_{3}}\left(t_{k}\right)=0.5 \cdot \frac{1}{t^{2}} \cdot\left(1-\mathrm{e}^{-2 t_{k}^{2}}\right)-\frac{1}{t^{4}} \cdot\left(1-\mathrm{e}^{-t_{k}^{2}}\right)^{2} .
\end{aligned}
$$

These functions are plotted in Fig. 2, page 5, with three different sampling strategies for $t_{k} \in[0,10]$.

- In the upper figure (2.a), we propose a suited sampling rate, $t_{k}=0.01 \cdot k$. A first conclusion that can be drawn from this figure is that even in a best case scenario $p_{3}$ is a poor-sensitive parameter as $V_{T_{3}}\left(t_{k}\right)<0.1$ and $S_{T_{3}}\left(t_{k}\right)<0.2$.

- In the middle figure (2.b), we choose measurement time instants so as to get colinear total variance functions, $V_{T_{1}}\left(t_{k}\right)$ and $V_{T_{2}}\left(t_{k}\right)$. For such sampling instants $\sin \left(2 \pi t_{k}\right)^{2}=1 / 4$, and $y$ becomes

$$
y\left(t_{k}, \mathbf{p}\right)=\left(2 p_{1} \pm 2 p_{2}\right) \cdot \mathrm{e}^{-0.1 t_{k}}+\mathrm{e}^{-p_{3} t_{k}^{2}}
$$

indicating the non-identifiablity of both $p_{1}$ and $p_{2}$.

- The bottom figure (2.c) exploits the total variance function for $t_{k}=0.5 \cdot k$. In this case, for all $t_{k}$, the total variance $V_{T_{2}}\left(t_{k}\right)$ is null, leaving only one sensitive parameter: $p_{1}$.

This example illustrates that experimental factors as measurement sampling rates may cause a lack of parameter identifiability in practice. This latter was detected by null (or inferior to an empirical threshold) total variance functions.

Remark The converse implication of the Prop. 4.2 is false: a non-identifiable parameter can have a non null total sensitivity function. This is described in Fig. 3 by the dotted arrow $\mathbf{1}$.

\subsection{Case (b): non-injectivity of the $\Psi$-functions}

Let us consider a not null $\Psi_{i}$ function, non-injective w.r.t. parameter $p_{i}$. In this context, the following proposition can be formulated :

Proposition 4.3 The non-injectivity w.r.t. $p_{i}$ of the function $\Psi_{i}\left(t_{k}, \mathbf{p}\right)$ (independently of $\left.t_{k}\right)$, implies the non-identifiability of the parameter $p_{i}$.

Proof Let $\mathbf{p}_{\sim i}$ be fixed, with $\mathbf{p}_{\sim i}$ the vector composed by all the parameters except $p_{i}$. As $\Psi_{i}\left(t_{k}, \mathbf{p}\right)$ is a noninjective function w.r.t. parameter $p_{i}$, there exists $p_{i} \neq$ $p_{i}^{*}$ such that,

$$
\Psi_{i}\left(t_{k}, \mathbf{p}_{\sim i}, p_{i}\right)=\Psi_{i}\left(t_{k}, \mathbf{p}_{\sim i}, p_{i}^{*}\right), \forall t_{k} \in \mathbb{T} .
$$

Therefore, setting $\mathbf{p}=\left[\mathbf{p}_{\sim i}, p_{i}\right] \in \mathbb{P}$ and $\mathbf{p}^{*}=\left[\mathbf{p}_{\sim i}, p_{i}^{*}\right] \in$ $\mathbb{P}$, it follows that

$$
\begin{aligned}
y\left(t_{k}, \mathbf{p}\right) & =y_{0}\left(t_{k}\right)+\Psi_{i}\left(t_{k}, \mathbf{p}\right)+\Psi_{\sim i}\left(t_{k}, \mathbf{p}_{\sim i}\right) \\
& =y_{0}\left(t_{k}\right)+\Psi_{i}\left(t_{k}, \mathbf{p}^{*}\right)+\Psi_{\sim i}\left(t_{k}, \mathbf{p}_{\sim i}^{*}\right) \\
& =y\left(t_{k}, \mathbf{p}^{*}\right)
\end{aligned}
$$

hence the non-identifiability of the parameter $p_{i}$.

\section{Example 2}

Let us consider the following model,

$y\left(t_{k}, \mathbf{p}\right)=\left(p_{3}^{2}-2 p_{2} p_{3}+\frac{1}{4}\right) \cdot\left(1-\mathrm{e}^{-t_{k}}\right)+p_{1}\left(1-\mathrm{e}^{-t_{k} p_{2}}\right)$

whereas the $\Psi_{3}$ function can be written as

$$
\Psi_{3}\left(t_{k}, \mathbf{p}\right)=\left(p_{3}^{2}-2 p_{2} p_{3}+p_{2}-\frac{1}{3}\right) \cdot\left(1-\mathrm{e}^{-t_{k}}\right) .
$$

Thus, $y\left(t_{k}, \mathbf{p}\right)$, respectively $\Psi_{3}\left(t_{k}, \mathbf{p}\right)$, are both noninjective w.r.t. parameter $p_{3}$ since, for all $t_{k} \in \mathbb{T}$ we can write

$$
\begin{gathered}
y\left(t_{k}, p_{1}, p_{2}, p_{3}\right)=y\left(t_{k}, p_{1}, p_{2}, 2 p_{2}-p_{3}\right) \\
\Psi_{3}\left(t_{k}, p_{1}, p_{2}, p_{3}\right)=\Psi_{3}\left(t_{k}, p_{1}, p_{2}, 2 p_{2}-p_{3}\right) .
\end{gathered}
$$

It is worth pointing out that the parameters $p_{1}$ and $p_{2}$ are globally identifiable.

Remark The converse implication of the Prop. 4.3, represented in Fig. 3 by the dotted arrow $\mathbf{2}$, is false: a nonidentifiable parameter can have an injective $\Psi_{i}$ function. Indeed the lack of identifiability can be due to different causes and, among others, to the insensitivity of the model output with respect to parameters as emphasized in section 4.2 .

Limit 1: This remark raises another question about the link of a non-injective output with respect to a parameter and the (total) sensitivity functions relative to the parameter in question. The total variance w.r.t. the parameter $p_{i}$ is $V_{T_{i}}\left(t_{k}\right)=\int_{\mathbf{I}^{n}} \Psi_{i}^{2}\left(t_{k}, \mathbf{p}\right) \mathrm{d} \mathbf{p}$. The integral operator prevents to detect the non-injectivity of $\Psi$-functions from the analysis of the associated sensitivity functions. This first limit about the use of the variance-based global sensitivity approaches to address non-identifiability questions is described in Fig. 3 by the dotted arrow $\mathbf{L}_{\mathbf{1}}$.

\subsection{Case (c): colinear sensitivity functions}

If the connection between a null sensitivity function $S_{T_{i}}$ and the non-identifiability of the parameter $p_{i}$ seems 
quite straightforward, the link between colinear sensitivity functions and parameter non-identifiability is less obvious. We illustrate herein a class of colinear sensitivity functions related to non-identifiable parameters, more precisely when two $\Psi_{i}$ and $\Psi_{j}$ functions are colinear w.r.t. time.

Throughout this subsection, we will explain the different facets of the presented problem through the use of a simple example introduced below.

\section{Example 3}

Consider the following academic example

$$
y\left(t_{k}, \mathbf{p}\right)=p_{2} \cdot p_{3} \cdot\left(1-\mathrm{e}^{-t_{k}}\right)+p_{1} \cdot p_{4} \cdot\left(1-\mathrm{e}^{-t_{k} \cdot p_{2}}\right)
$$

with $\mathbf{p} \in[0,1]^{4}$. In this case, the parameters $p_{1}$ and $p_{4}$ can not be mutually (a priori globally) identifiable (see section 4.4.3) and the sensitivity study reveals two colinear sensitivity functions, $S_{T_{1}}$ and $S_{T_{4}}$ (see section 4.4.4).

\subsubsection{Definition of $\Omega$-functions}

The compensation effects study of two parameters, $p_{i}$ and $p_{j}$, involves the use of the $\Psi_{i}$ and $\Psi_{j}$ functions, which are further decomposed as

$$
\begin{aligned}
& \Psi_{i}\left(t_{k}, \mathbf{p}\right)=\Omega_{i}\left(t_{k}, \mathbf{p}_{\sim j}\right)+\Omega_{i, j}\left(t_{k}, \mathbf{p}\right) \\
& \Psi_{j}\left(t_{k}, \mathbf{p}\right)=\Omega_{j}\left(t_{k}, \mathbf{p}_{\sim i}\right)+\Omega_{i, j}\left(t_{k}, \mathbf{p}\right)
\end{aligned}
$$

where $i \neq j$, and

$\Omega_{i}$ represents the influence on the output $y$ of the parameter $p_{i}$ independently of $p_{j}$; taking into account the property $\left(\mathcal{P}_{2}\right)$ of the decomposition (3), $\Omega_{i}\left(t, \mathbf{p}_{\sim j}\right)$ can be also defined as

$$
\Omega_{i}\left(t_{k}, \mathbf{p}_{\sim j}\right)=\int_{0}^{1} \Psi_{i}\left(t_{k}, \mathbf{p}\right) d p_{j}
$$

$\Omega_{i, j}$ is its complementary effect on $y$ (corresponding to the combined action on $y$ of $p_{i}$ and $\left.p_{j}\right)$, i.e. ${ }^{3}$

$$
\Omega_{i, j}\left(t_{k}, \mathbf{p}\right)=\Psi_{i}\left(t_{k}, \mathbf{p}\right)-\Omega_{i}\left(t_{k}, \mathbf{p}_{\sim j}\right)
$$

We address here only the time dependence between only two $\Psi$ functions, but it could be further developed in

\footnotetext{
3 Our main aim is to express the compensation on the model output $y$ of the effects of parameters $p_{i}$ and $p_{j}$. As the two $\Psi$-functions have a common part, which depends mutually on the two parameters in question, we have decomposed $\Psi$ as the sum of two complementary $\Omega$-functions.
}

order to consider the compensation on the model output of more than two parameters.

In the case of $\Omega$-functions, we have the following properties, for $l \in\{i, j\}$,

$$
\begin{aligned}
& \int_{0}^{1} \Omega_{i}\left(t_{k}, \mathbf{p}_{\sim j}\right) d p_{i}=0 ; \quad \int_{0}^{1} \Omega_{j}\left(t_{k}, \mathbf{p}_{\sim i}\right) d p_{j}=0 \\
& \int_{0}^{1} \Omega_{i, j}\left(t_{k}, \mathbf{p}\right) d p_{l}=0
\end{aligned}
$$

i.e. the three $\Omega$-functions are orthogonal with respect to the parameters $p_{i}$ and $p_{j}$ (due to the orthogonality of the Sobol' decomposition terms in eq. (3)).

\subsubsection{On the linear dependence of $\Omega$-functions}

We are interested in the description of the consequences of the time dependency of two distinct $\Psi$ functions, on parameter non-identifiability and sensitivity colinearity.

Remark Considering three $\Omega$-functions: $\Omega_{i}, \Omega_{j}$ and $\Omega_{i, j}$, for reasons of simplicity we shall say "dependence", but in fact we mean the pairwise linear dependence of these functions.

Proposition 4.4 The time dependence of the functions $\Omega_{i}\left(t_{k}, \mathbf{p}_{\sim j}\right), \Omega_{i, j}\left(t_{k}, \mathbf{p}\right)$ and $\Omega_{j}\left(t_{k}, \mathbf{p}_{\sim i}\right)$, implies that they can be factorized as

$$
\begin{aligned}
& \Omega_{i}\left(t_{k}, \mathbf{p}_{\sim j}\right)=h_{i}\left(\mathbf{p}_{\sim j}\right) \cdot g\left(t_{k}, \mathbf{p}_{\sim i, j}\right) \\
& \Omega_{j}\left(t_{k}, \mathbf{p}_{\sim i}\right)=h_{j}\left(\mathbf{p}_{\sim i}\right) \cdot g\left(t_{k}, \mathbf{p}_{\sim i, j}\right) \\
& \Omega_{i, j}\left(t_{k}, \mathbf{p}\right)=h_{i, j}(\mathbf{p}) \cdot g\left(t_{k}, \mathbf{p}_{\sim i, j}\right) .
\end{aligned}
$$

Proof Let us consider the linear dependence, with respect to time, of the functions $\Omega_{i}\left(t_{k}, \mathbf{p}_{\sim j}\right)$ and $\Omega_{j}\left(t_{k}, \mathbf{p}_{\sim i}\right)$

$c_{i}(\mathbf{p}) \cdot \Omega_{i}\left(t_{k}, \mathbf{p}_{\sim j}\right)+c_{j}(\mathbf{p}) \cdot \Omega_{j}\left(t_{k}, \mathbf{p}_{\sim i}\right)=0, \forall t_{k} \in \mathbb{T}$

where $c_{i}(\mathbf{p})$ and $c_{j}(\mathbf{p})$ are non-null coefficients (parameter functions). Based on this equation, we can write the relationship between the $\Omega_{i}\left(t_{k}, \mathbf{p}_{\sim j}\right)$ and $\Omega_{j}\left(t_{k}, \mathbf{p}_{\sim i}\right)$ functions :

$\Omega_{i}\left(t_{k}, \mathbf{p}_{\sim j}\right)=-\frac{c_{j}(\mathbf{p})}{c_{i}(\mathbf{p})} \cdot \Omega_{j}\left(t_{k}, \mathbf{p}_{\sim i}\right)$
$\Omega_{j}\left(t_{k}, \mathbf{p}_{\sim i}\right)=-\frac{c_{i}(\mathbf{p})}{c_{j}(\mathbf{p})} \cdot \Omega_{i}\left(t_{k}, \mathbf{p}_{\sim j}\right)$

By fixing $p_{j}=0$ in (17), we can express $\Omega_{i}$ as

$\Omega_{i}\left(t_{k}, \mathbf{p}_{\sim j}\right)=-\frac{c_{j}\left(\mathbf{p}_{\sim j}, p_{j}=0\right)}{c_{i}\left(\mathbf{p}_{\sim j}, p_{j}=0\right)} \cdot \Omega_{j}\left(t_{k}, \mathbf{p}_{\sim i, j}, p_{j}=0\right)$ 
and furthermore as

$$
\Omega_{i}\left(t_{k}, \mathbf{p}_{\sim j}\right)=h_{i}\left(\mathbf{p}_{\sim j}\right) \cdot g\left(t_{k}, \mathbf{p}_{\sim i, j}\right)
$$

with $g\left(t_{k}, \mathbf{p}_{\sim i, j}\right)=\Omega_{j}\left(t_{k}, \mathbf{p}_{\sim i, j}, p_{j}=0\right)$ and $h_{i}\left(\mathbf{p}_{\sim j}\right)=$ $-c_{j}\left(\mathbf{p}_{\sim j}, p_{j}=0\right) / c_{i}\left(\mathbf{p}_{\sim j}, p_{j}=0\right)$.

Based on (18) and (19), and by fixing $p_{i}=0, \Omega_{j}$ can be written as

$$
\Omega_{j}\left(t_{k}, \mathbf{p}_{\sim i}\right)=h_{j}\left(\mathbf{p}_{\sim i}\right) \cdot g\left(t_{k}, \mathbf{p}_{\sim i, j}\right)
$$

with $h_{j}\left(\mathbf{p}_{\sim i}\right)$ defined as

$$
h_{j}\left(\mathbf{p}_{\sim i}\right)=-\frac{c_{i}\left(\mathbf{p}_{\sim i}, p_{i}=0\right) \cdot h_{i}\left(\mathbf{p}_{\sim i, j}, p_{i}=0\right)}{c_{j}\left(\mathbf{p}_{\sim i}, p_{i}=0\right)} .
$$

Let us now consider the linear dependence, w.r.t. time, of the functions $\Omega_{i}\left(t_{k}, \mathbf{p}_{\sim j}\right)$ (or equivalently $\Omega_{j}\left(t_{k}, \mathbf{p}_{\sim i}\right)$ ) and $\Omega_{i, j}\left(t_{k}, \mathbf{p}\right)$

$d_{i}(\mathbf{p}) \cdot \Omega_{i}\left(t_{k}, \mathbf{p}_{\sim j}\right)+d_{i, j}(\mathbf{p}) \cdot \Omega_{i, j}\left(t_{k}, \mathbf{p}\right)=0, \forall t_{k} \in \mathbb{T}$

where $d_{i}(\mathbf{p})$ and $d_{i, j}(\mathbf{p})$ are two non-null coefficients (parameter functions). Taking into account the factorization of the $\Omega_{i}\left(t_{k}, \mathbf{p}_{\sim j}\right)$ function in (19), we can write

$$
\Omega_{i, j}\left(t_{k}, \mathbf{p}\right)=-\frac{d_{i}(\mathbf{p}) \cdot h_{i}\left(\mathbf{p}_{\sim j}\right)}{d_{i, j}(\mathbf{p})} \cdot g\left(t_{k}, \mathbf{p}_{\sim i, j}\right),
$$

that is to say, the factorization of the $\Omega_{i, j}$ function proposed in the Prop. 4.4, with $h_{i, j}(\mathbf{p})$ defined by

$$
h_{i, j}(\mathbf{p})=-\frac{d_{i}(\mathbf{p}) \cdot h_{i}\left(\mathbf{p}_{\sim j}\right)}{d_{i, j}(\mathbf{p})} .
$$

Remark If we further assume that the functions $\Omega_{i}\left(t_{k}, \mathbf{p}_{\sim j}\right), \Omega_{j}\left(t_{k}, \mathbf{p}_{\sim i}\right)$ and $\Omega_{i, j}\left(t_{k}, \mathbf{p}\right)$ are also $\mathbf{p}_{\sim i, j}$ dependent, then, the same pattern of proof implies that:

$$
\begin{aligned}
& \Omega_{i}\left(t_{k}, \mathbf{p}_{\sim j}\right)=h_{i}\left(p_{i}\right) \cdot g\left(t_{k}, \mathbf{p}_{\sim i, j}\right) \\
& \Omega_{j}\left(t_{k}, \mathbf{p}_{\sim i}\right)=h_{j}\left(p_{j}\right) \cdot g\left(t_{k}, \mathbf{p}_{\sim i, j}\right) \\
& \Omega_{i, j}\left(t_{k}, \mathbf{p}\right)=h_{i, j}\left(p_{i}, p_{j}\right) \cdot g\left(t_{k}, \mathbf{p}_{\sim i, j}\right) .
\end{aligned}
$$

This is a stronger condition than the time dependence of $\Omega$-functions, and it will be employed to show the colinearity of sensitivity measures.

Example 3 (continued)

In order to analyze the compensation effects on the model output in (13) of the parameters $p_{1}$ and $p_{4}$, we must firstly construct the $\Psi$ functions

$$
\begin{aligned}
& \Psi_{1}\left(t_{k}, \mathbf{p}\right)=\frac{p_{4}\left(2 p_{1}-1\right)\left(1-\mathrm{e}^{-t_{k} \cdot p_{2}}\right)}{2} \\
& \Psi_{4}\left(t_{k}, \mathbf{p}\right)=\frac{p_{1}\left(2 p_{4}-1\right)\left(1-\mathrm{e}^{-t_{k} p_{2}}\right)}{2}
\end{aligned}
$$

which can be further developed respectively as the sum of two complementary $\Omega$-functions

$$
\begin{aligned}
\Omega_{1}\left(t_{k}, \mathbf{p}_{\sim 4}\right) & =\frac{\left(2 p_{1}-1\right)\left(1-\mathrm{e}^{-t_{k} p_{2}}\right)}{4} \\
\Omega_{4}\left(t_{k}, \mathbf{p}_{\sim 1}\right) & =\frac{\left(2 p_{4}-1\right)\left(1-\mathrm{e}^{-t_{k} p_{2}}\right)}{4} \\
\Omega_{1,4}\left(t_{k}, \mathbf{p}\right) & =\frac{\left(2 p_{1}-1\right)\left(2 p_{4}-1\right)\left(1-\mathrm{e}^{-t_{k} p_{2}}\right)}{4} .
\end{aligned}
$$

As it can be observed, the functions $\Omega_{1}, \Omega_{4}$ and $\Omega_{1,4}$, are time and $\mathbf{p}_{\sim 1,4}$ dependent, and furthermore they can be factorized as seen in eq. (21).

\subsubsection{On the time linear dependence of $\Omega$-functions and non-identifiability of $p_{i}$ or $p_{j}$}

The consequence of the time linear dependence of $\Omega$ functions on the parameter non-identifiability is stated below.

Proposition 4.5 The linear dependence, w.r.t. time, of the functions $\Omega_{i}\left(t_{k}, \mathbf{p}_{\sim j}\right), \Omega_{i, j}\left(t_{k}, \mathbf{p}\right)$ and $\Omega_{j}\left(t_{k}, \mathbf{p}_{\sim i}\right)$ implies the non-identifiability of $p_{i}$ or $p_{j}$.

Proof Let us write $y\left(t_{k}, \mathbf{p}\right)$ as

$$
\begin{aligned}
y\left(t_{k}, \mathbf{p}\right)= & y_{0}\left(t_{k}\right)+\Omega_{i}\left(t_{k}, \mathbf{p}_{\sim j}\right)+\Omega_{i, j}\left(t_{k}, \mathbf{p}\right)+ \\
& +\Omega_{j}\left(t_{k}, \mathbf{p}_{\sim i}\right)+\Psi_{\sim i, j}\left(t_{k}, \mathbf{p}_{\sim i, j}\right)
\end{aligned}
$$

where the function $\Psi_{\sim i, j}$ regroups all the terms in Sobol' expansion involving the parameters $\mathbf{p}_{\sim i, j}$. The time dependence of the functions $\Omega_{i}\left(t_{k}, \mathbf{p}_{\sim j}\right), \Omega_{i, j}\left(t_{k}, \mathbf{p}\right)$ and $\Omega_{j}\left(t_{k}, \mathbf{p}_{\sim i}\right)$ implies the factorisation of these functions, as seen in Proposition 4.4. Then, we can write $y\left(t_{k}, \mathbf{p}\right)$ as

$y\left(t_{k}, \mathbf{p}\right)=y_{0}\left(t_{k}\right)+h(\mathbf{p}) \cdot g\left(t, \mathbf{p}_{\sim i, j}\right)+\Psi_{\sim i, j}\left(t_{k}, \mathbf{p}_{\sim i, j}\right)$

with $h(\mathbf{p})=h_{i}\left(\mathbf{p}_{\sim j}\right)+h_{j}\left(\mathbf{p}_{\sim i}\right)+h_{i, j}(\mathbf{p})$. Hence, for almost all $\mathbf{p}^{*}=\left[\mathbf{p}_{\sim i, j}, p_{i}^{*}, p_{j}^{*}\right] \in \mathbb{P}$,

$$
y\left(t_{k}, \mathbf{p}\right)=y\left(t_{k}, \mathbf{p}^{*}\right) \forall t_{k} \in \mathbb{T},
$$

implies that $h(\mathbf{p})=h\left(\mathbf{p}^{*}\right)$, i.e., one equation with two unknown parameters, and an infinity of possible $\left(p_{i}, p_{j}\right)$ solutions. As a consequence, it is impossible to estimate both $p_{i}$ and $p_{j}$. 


\section{Example 3 (continued)}

Since $\Omega_{1}, \Omega_{4}$ and $\Omega_{1,4}$, defined in (24)-(26), are linearly dependent w.r.t. time, from Prop. $4.5, p_{1}$ and $p_{4}$ are mutually non-identifiable. This fact could also be seen through a Taylor series approach as stated below.

Considering the model expressed in (13), the successive derivatives of $y$ at $t_{0}=0^{+}$satisfy $a_{0}(\mathbf{p})=0, a_{1}(\mathbf{p})=$ $p_{2} p_{3}+p_{1} p_{4} p_{2}, a_{2}(\mathbf{p})=-p_{2} p_{3}-p_{1} p_{4} p_{2}^{2}$ etc. It is easy to show that

$$
a_{k}(\hat{\mathbf{p}})=a_{k}\left(\mathbf{p}^{*}\right), k=1,2, \ldots, 5
$$

has two solutions for $\hat{\mathbf{p}}$, namely $\hat{\mathbf{p}}^{1}=\left(p_{1}^{*}, p_{2}^{*}, p_{3}^{*}, p_{4}^{*}\right)$ and $\hat{\mathbf{p}}^{2}=\left(p_{4}^{*}, p_{2}^{*}, p_{3}^{*}, p_{1}^{*}\right)$.

The second and the third parameter, which take the same values in the two solutions, are globally identifiable. The other two, which can each take two values, are only locally identifiable.

\subsubsection{On the time linear dependence of $\Omega$-functions and total sensitivity functions}

Proposition 4.6 Assume that for all $t_{k} \in \mathbb{T}$, the functions $\Omega_{i}\left(t_{k}, \mathbf{p}_{\sim j}\right), \Omega_{i, j}\left(t_{k}, \mathbf{p}\right)$ and $\Omega_{j}\left(t_{k}, \mathbf{p}_{\sim i}\right)$ are time and $\mathbf{p}_{\sim i, j}$ dependent. Then, the total sensitivity functions $V_{T_{i}}\left(t_{k}\right)$ and $V_{T_{j}}\left(t_{k}\right)$ (defined in Section 4.1) are colinear.

Proof Given Proposition 4.4 and the definition of the functions $\Psi_{i}$ and $\Psi_{j}$, we can write:

$$
\begin{aligned}
& \Psi_{i}\left(t_{k}, \mathbf{p}\right)=\left(h_{i}\left(p_{i}\right)+h_{i, j}\left(p_{i}, p_{j}\right)\right) \cdot g\left(t_{k}, \mathbf{p}_{\sim i, j}\right) \\
& \Psi_{j}\left(t_{k}, \mathbf{p}\right)=\left(h_{j}\left(p_{j}\right)+h_{i, j}\left(p_{i}, p_{j}\right)\right) \cdot g\left(t_{k}, \mathbf{p}_{\sim i, j}\right) .
\end{aligned}
$$

Total variance functions can thus be written as

$$
\begin{aligned}
V_{T_{i}}\left(t_{k}\right)= & \left(\int_{0}^{1} h_{i}^{2}\left(p_{i}\right) d p_{i}+\int_{\mathbf{I}^{2}} h_{i, j}^{2}\left(p_{i}, p_{j}\right) d \mathbf{p}_{i, j}\right) . \\
& \cdot \int_{\mathbf{I}^{n-2}} g^{2}\left(t_{k}, \mathbf{p}_{\sim i, j}\right) d \mathbf{p}_{\sim i, j} \\
V_{T_{j}}\left(t_{k}\right)= & \left(\int_{0}^{1} h_{j}^{2}\left(p_{j}\right) d p_{j}+\int_{\mathbf{I}^{2}} h_{i, j}^{2}\left(p_{i}, p_{j}\right) d \mathbf{p}_{i, j}\right) . \\
& \cdot \int_{\mathbf{I}^{n-2}} g^{2}\left(t_{k}, \mathbf{p}_{\sim i, j}\right) d \mathbf{p}_{\sim i, j} .
\end{aligned}
$$

It is clear that $V_{T_{i}}\left(t_{k}\right)$ and $V_{T_{j}}\left(t_{k}\right)$ are time-colinear. Furthermore, as $V\left(t_{k}\right)$ is a non-null function, this directly implies the colinearity of the total sensitivity functions $S_{T_{1}}\left(t_{k}\right)$ and $S_{T_{2}}\left(t_{k}\right)$ as well.

Remark By integrating over the parameters $\mathbf{p}_{\sim i, j}$ the equation of (time and $\mathbf{p}_{\sim i, j}$ ) dependence between $\Omega_{i}\left(t_{k}, \mathbf{p}_{\sim j}\right)$ and $\Omega_{j}\left(t_{k}, \mathbf{p}_{\sim i}\right)$

$$
\alpha_{i}\left(p_{i}, p_{j}\right) \cdot \Omega_{i}\left(t_{k}, \mathbf{p}_{\sim j}\right)+\alpha_{j}\left(p_{i}, p_{j}\right) \cdot \Omega_{j}\left(t_{k}, \mathbf{p}_{\sim i}\right)=0
$$

we obtain the linear dependence w.r.t. time of $y_{i}$ and $y_{j}$ functions

$$
\alpha_{i}\left(p_{i}, p_{j}\right) \cdot y_{i}\left(t_{k}, p_{i}\right)+\alpha_{j}\left(p_{i}, p_{j}\right) \cdot y_{j}\left(t_{k}, p_{j}\right)=0
$$

as

$$
\begin{aligned}
& \int_{\mathbf{I}^{n-2}} \Omega_{i}\left(t_{k}, \mathbf{p}_{\sim j}\right) d \mathbf{p}_{\sim i, j}=y_{i}\left(t_{k}, p_{i}\right) \\
& \int_{\mathbf{I}^{n-2}} \Omega_{j}\left(t_{k}, \mathbf{p}_{\sim j}\right) d \mathbf{p}_{\sim i, j}=y_{j}\left(t_{k}, p_{j}\right)
\end{aligned}
$$

due to the property $\left(\mathcal{P}_{2}\right)$ of the Sobol' decomposition (3). We can then conclude (by the same pattern of proof as in Prop. 4.4 and 4.6) to the colinearity of the first-order variance functions, $V_{i}$ and $V_{j}$ (as defined in (4)).

\section{Example 3 (continued)}

Let us continue the analysis of the model defined in (13). Firstly, recall that the functions $\Omega_{1}, \Omega_{1,4}$ and $\Omega_{4}$ are linear dependent w.r.t. time as emphasized in (24)-(26). The first-order and total variances of the model output w.r.t. the parameters $p_{1}$ and $p_{4}$ are defined as

$$
\begin{aligned}
& V_{1}\left(t_{k}\right)=V_{4}\left(t_{k}\right)=\frac{\left(1-t_{k}-\mathrm{e}^{-t_{k}}\right)^{2}}{48 \cdot t_{k}^{2}} \\
& V_{T_{1}}\left(t_{k}\right)=V_{T_{4}}\left(t_{k}\right)=\frac{4 \cdot \mathrm{e}^{-t_{k}}-\mathrm{e}^{-2 t_{k}}+2 \cdot t_{k}-3}{72 \cdot t_{k}}
\end{aligned}
$$

whereas the second-order variance function $V_{1,4}\left(t_{k}\right)$ is

$$
V_{1,4}\left(t_{k}\right)=\frac{\left(1-t_{k}-\mathrm{e}^{-t_{k}}\right)^{2}}{48 \cdot t_{k}^{2}}
$$

This example shows that time and $\mathbf{p}_{\sim i, j}$ dependent $\Omega$ functions lead to colinear (first-order and total) sensitivity functions.

Limit 2: Conversely to the general idea in local identifiability analysis - where the non-identifiable parameters correspond to null or colinear local sensitivity functions - in global sensitivity analysis colinear sensitivity functions will not lead necessarily to parameter nonidentifiability. Indeed, the converse of the Proposition 4.6 is not always true. This second limit, noted $\mathbf{L}_{\mathbf{2}}$ in Fig. 3, prevents to conclude surely on non-identifiability from the colinear analysis of sensitivity functions. Hereafter, we give two counterexamples in order to illustrate these cases.

\section{Counterexample 1}

Let us consider the two parameter model represented by

$$
y\left(t_{k}, \mathbf{p}\right)=\mathrm{e}^{-\left(p_{1}+p_{2}\right) \cdot t_{k}}
$$

with $\mathbf{p} \in[0,1]^{2}$ and the following Sobol' decomposition $y\left(t_{k}, \mathbf{p}\right)=y_{0}\left(t_{k}\right)+y_{1}\left(t_{k}, p_{1}\right)+y_{2}\left(t_{k}, p_{2}\right)+y_{1,2}\left(t_{k}, p_{1}, p_{2}\right)$. 
Furthermore, the $\Omega$-functions, as defined in section 4.4.1, can be expressed as

$$
\begin{aligned}
\Omega_{1}\left(t_{k}, \mathbf{p}_{\sim 2}\right)= & y_{1}\left(t_{k}, p_{1}\right)=\frac{\left(t_{k} \mathrm{e}^{-p_{1} t_{k}}-1+\mathrm{e}^{-t_{k}}\right)\left(1-\mathrm{e}^{-t_{k}}\right)}{t_{k}^{2}} \\
\Omega_{2}\left(t_{k}, \mathbf{p}_{\sim 1}\right)= & y_{2}\left(t_{k}, p_{2}\right)=\frac{\left(t_{k} \mathrm{e}^{-p_{2} t_{k}}-1+\mathrm{e}^{-t_{k}}\right)\left(1-\mathrm{e}^{-t_{k}}\right)}{t_{k}^{2}} \\
\Omega_{1,2}\left(t_{k}, \mathbf{p}\right)= & y_{1,2}\left(t_{k}, p_{1}, p_{2}\right)=\mathrm{e}^{-\left(p_{1}+p_{2}\right) \cdot t_{k}} \\
& -\frac{\left(t_{k} \mathrm{e}^{-p_{1} t_{k}}+t_{k} \mathrm{e}^{-p_{2} t_{k}}-1+\mathrm{e}^{-t_{k}}\right)\left(1-\mathrm{e}^{-t_{k}}\right)}{t_{k}^{2}}
\end{aligned}
$$

whereas the variance functions becomes

$$
\begin{aligned}
& V_{1}\left(t_{k}\right)=\frac{\left(1-\mathrm{e}^{-t_{k}}\right)^{3}\left(-2+2 \mathrm{e}^{-t_{k}}+t_{k}+t_{k} \mathrm{e}^{-t_{k}}\right)}{2 t_{k}^{4}} \\
& V_{T_{1}}\left(t_{k}\right)=\frac{\left(1-\mathrm{e}^{-t_{k}}\right)^{2}\left(1+\mathrm{e}^{-t_{k}}\right)\left(t_{k}+t_{k} \mathrm{e}^{-t_{k}}+2 \mathrm{e}^{-t_{k}}-2\right)}{4 t_{k}^{3}} .
\end{aligned}
$$

with $V_{2}\left(t_{k}\right)=V_{1}\left(t_{k}\right)$ and $V_{T_{2}}\left(t_{k}\right)=V_{T_{1}}\left(t_{k}\right)$. As shown by previous equations, the functions $\Omega_{1}$ and $\Omega_{2}$ are not colinear despite the equality of variance functions. This counterexample shows that the colinearity of sensitivity functions has other causes than the time dependence of $\Omega$-functions.

\section{Counterexample 2}

Let us consider a two-parameter generic model decomposed as

$y\left(t_{k}, \mathbf{p}\right)=y_{0}\left(t_{k}\right)+y_{1}\left(t_{k}, p_{1}\right)+y_{2}\left(t_{k}, p_{2}\right)+y_{1,2}\left(t_{k}, \mathbf{p}\right)$.

We denote by $V_{1}\left(t_{k}\right)=\int_{0}^{1} y_{1}^{2}\left(t_{k}, p_{1}\right) d p_{1}, V_{2}\left(t_{k}\right)=$ $\int_{0}^{1} y_{2}^{2}\left(t_{k}, p_{2}\right) d p_{2}$ and $V_{1,2}\left(t_{k}\right)=\int_{\mathbf{I}^{2}} y_{1,2}^{2}\left(t_{k}, p_{1}, p_{2}\right) d \mathbf{p}$, the first-order, respectively second-order variance functions. Consider the following modified model

$$
\tilde{y}\left(t_{k}, \mathbf{p}\right)=\frac{y_{1}\left(t_{k}, p_{1}\right)}{\sqrt{V_{1}\left(t_{k}\right)}}+\frac{y_{2}\left(t_{k}, p_{2}\right)}{\sqrt{V_{2}\left(t_{k}\right)}}+\frac{y_{1,2}\left(t_{k}, \mathbf{p}\right)}{\sqrt{V_{1,2}\left(t_{k}\right)}} .
$$

In this case, for non-null functions $y_{1}, y_{2}$ and $y_{1,2}$, we obtain the following variances:

$$
\tilde{V}_{1}\left(t_{k}\right)=1=\tilde{V}_{2}\left(t_{k}\right)=\tilde{V}_{1,2}\left(t_{k}\right)
$$

and

$$
\tilde{V}_{T_{1}}\left(t_{k}\right)=2=\tilde{V}_{T_{2}}\left(t_{k}\right) .
$$

whereas the $\tilde{\Omega}$-functions, defined in an equivalent manner as previously, are not necessarily linear dependent w.r.t. time.

For example, let us consider the following model with two globally identifiable parameters

$$
y\left(t_{k}, \mathbf{p}\right)=2 p_{1}+\mathrm{e}^{p_{2} t_{k}}-\frac{\mathrm{e}^{t_{k}}-1}{t_{k}}-1
$$

having the following decomposition terms

$$
y_{1}\left(t_{k}, p_{1}\right)=2 p_{1}-1 ; y_{2}\left(t_{k}, p_{2}\right)=\mathrm{e}^{p_{2} t_{k}}-\frac{\mathrm{e}^{t_{k}}-1}{t_{k}}
$$

with null $y_{0}$ and $y_{1,2}$ functions. The modified model, constructed as in (28), will be composed into

$$
\tilde{y}_{1}\left(t_{k}, p_{1}\right)=\sqrt{3}\left(2 p_{1}-1\right) ; \tilde{y}_{2}\left(t_{k}, p_{2}\right)=\frac{\mathrm{e}^{p_{2} t_{k}}-\frac{\mathrm{e}^{t_{k}}-1}{t_{k}}}{\sqrt{V_{2}\left(t_{k}\right)}}
$$

with $V_{2}\left(t_{k}\right)=\frac{\mathrm{e}^{2 t_{k}}-1}{2 t_{k}}-\frac{\left(\mathrm{e}^{t_{k}}-1\right)^{2}}{t_{k}^{2}}$. As the $\tilde{y}_{1,2}$ function is null for all $t_{k} \in \mathbb{T}$, the $\Omega$-functions are expressed as: $\tilde{\Omega}_{1}\left(t, \mathbf{p}_{\sim 2}\right)=\tilde{y}_{1}\left(t, p_{1}\right), \tilde{\Omega}_{2}\left(t, \mathbf{p}_{\sim 1}\right)=\tilde{y}_{2}\left(t, p_{2}\right)$ and $\tilde{\Omega}_{1,2}(t, \mathbf{p})=0$. In this case, the three $\Omega$-functions are not time linearly dependent, despite the colinearity of variance functions as stressed in (29) and (30). This illustrates the fact that the converse of Prop. 4.6 is not always true and it proves that colinear (first-order and total) sensitivity functions could be associated also to identifiable parameters.

\subsection{Discussions}

\subsubsection{Summary of results}

Let us give a brief summary of the results concerning the connections between parameter non-identifiability and output sensitivity (illustrated in Fig. 3):

- the first relationship between the two notions corresponds to the generally acknowledged association between insensitive parameters, i.e., null total sensitivity functions, and non-identifiable parameters (expressed through propositions 4.1 and 4.2);

- secondly, it was shown that the non-injectivity of $\Psi$ functions also leads to the non-identifiability conclusion (Prop. 4.3);

- as shown in Propositions 4.4 to 4.6, the linear dependence w.r.t. time and $\mathbf{p}_{\sim i, j}$ of three $\Omega$-functions $\left(\Omega_{i}\right.$, $\Omega_{j}$ and $\left.\Omega_{i, j}\right)$ implies both the non-identifiability of the parameters in question (Prop. 4.5) and the colinearity of their total sensitivity functions (Prop. 4.6).

Fig. 3 emphasizes the central role played by the $\Omega$ and $\Psi$ functions in the relationships between identifiability and sensitivity.

\subsubsection{Limits of sensitivity analysis for inferring non- identifiability}

The converse of the proposition 4.1, represented by the arrow 4 in Fig. 3, is true, i.e. a null $\Psi_{i}$ function will also imply a null total sensitivity function, $S_{T_{i}}\left(t_{k}\right), \forall t_{k} \in \mathbb{T}$. 


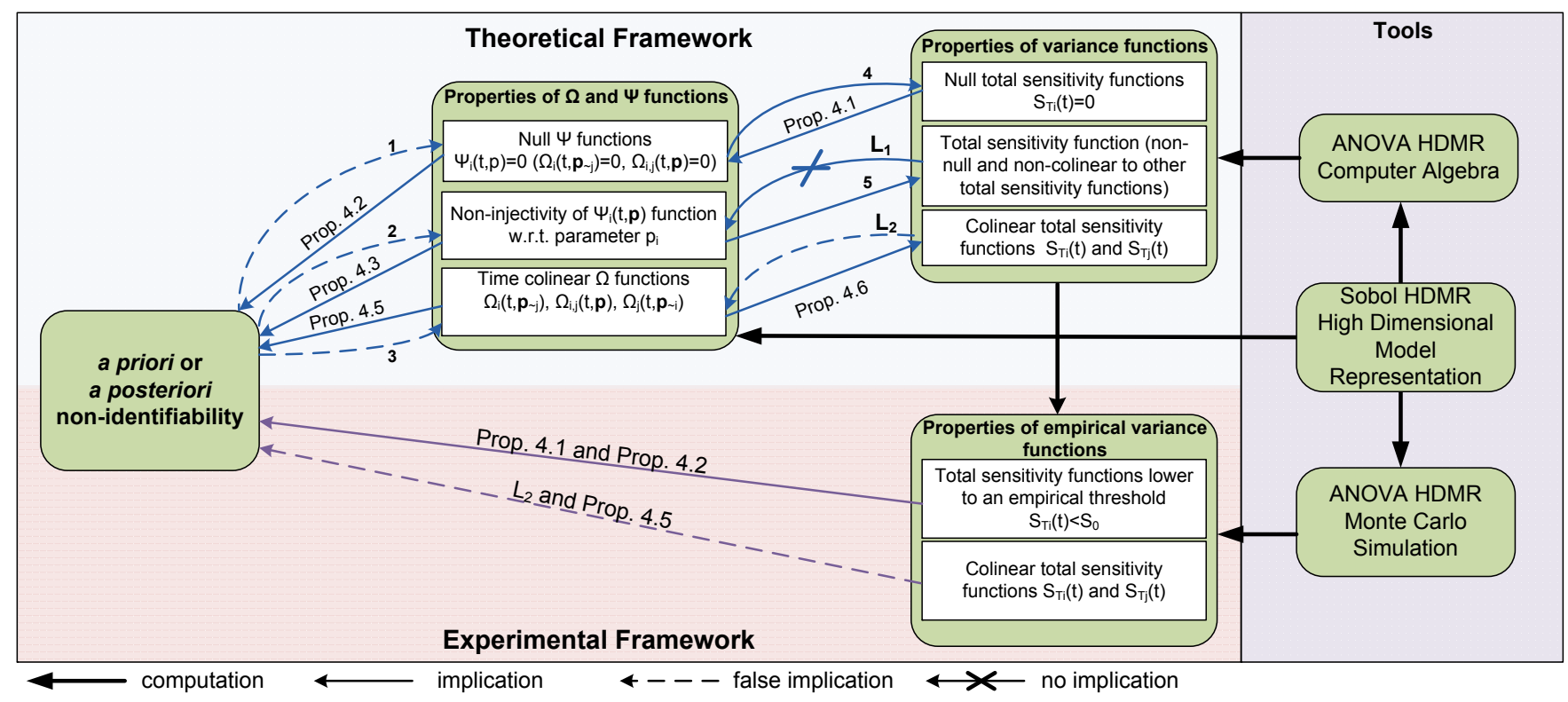

Fig. 3. Schematic representation of relationships between non-identifiability and sensitivity. The link between $\Psi$ or $\Omega$-functions and parameter non-identifiability is represented through propositions 4.2, 4.3 and 4.5, whereas their converses are false (represented by the dashed arrows $\mathbf{1}, \mathbf{2}, \mathbf{3}$ ). The link between $\Psi$ or $\Omega$-functions and variance or sensitivity functions is described through propositions 4.1 and 4.6 and the arrow $\mathbf{5}$ (this arrow represents the fact that for a non-injective function $\Psi$, we can compute its total variance with respect to the parameter in question). The false implication $\mathbf{L}_{\mathbf{1}}$, represents the fact that the non-injectivity of the model output w.r.t. a parameter can not be tested from global sensitivity measures. The converse of the proposition 4.1 (arrow 4) is true, while that of the proposition 4.6 is not always true (arrow $\mathbf{L}_{\mathbf{2}}$ ). In an experimental framework, a total sensitivity function inferior to an empirical threshold implies the non-identifiability of the parameter in question, whereas the colinearity of total (or first order) sensitivity function must be treated with caution, since it does not allow to conclude surely on the non-identifiability, conversely to what is admitted in local identifiability. The available tools for the computation of variance/sensitivity functions are represented on the right side of the figure.

However, the converse of Propositions 4.2, 4.3 and 4.5 are false (represented by the dashed arrows $\mathbf{1}, \mathbf{2}$ and $\mathbf{3}$ ). They only represent necessary identifiability conditions. Similarly, the converse of the Proposition 4.6 is not valid, since the colinearity of the (first-order and total) sensitivity functions can be caused by other sources than the linear dependence w.r.t. time of the $\Omega$-functions (as seen in the Counterexamples $\mathbf{1}$ and $\mathbf{2}$ ).

As summarized in Fig. 3, two limits, $\mathbf{L}_{\mathbf{1}}$ and $\mathbf{L}_{\mathbf{2}}$, prevent to conclude on global non-identifiability from the analysis of global sensitivity functions. The first one is due to the impossibility to test the non-injectivity from the variance functions. The second one shows that the colinearity analysis of sensitivity functions, usually employed in local identifiability analysis, is not always valid. Finally, testing low-sensitizing parameters associated with sensitivity functions lower to an empirical threshold is the only property that leads in practice to conclude surely on global a posteriori non-identifiability.

\section{Conclusion}

The lack of available methods to test the global a posteriori identifiability of parameters in high dimensional dynamic models probably explains the success of global sensitivity techniques, particulary in systems biology and more precisely in the identification of metabolic pathways. Nevertheless, the links between these two analyses are not yet fully explored. This present work provides new insights into the relationships between them. We show that the lack of identifiability may be due to three causes: insensitivity, colinearity, or the non-injectivity of the functions involved in the global sensitivity analysis. While the first is in truth a general acknowledged association, between insensitive parameters and non-identifiable parameters, the other two are less straightforward. Indeed, these two points correspond to two limits which prevent a sure conclusion on global non-identifiability. The first limit is due to the impossibility of testing the non-injectivity from the variance functions. The second shows that, conversely to what is admitted in local identifiability, colinear analysis of sensitivity functions is no longer valid. Thus, the conclusions about parameter non-identifiability drawn from the analysis of global sensitivity functions must be carefully analyzed.

This study also brings out the central role of some functions, entitled $\Omega$ and $\Psi$ functions, in both sensitivity and identifiability analyses. These functions are derived from Sobol's high dimensional representation of the model 
output. They could be regarded as a promising perspective to solve the global identifiability issue in practice. If the explicit expression of the output variable is known, those functions can be determined by computer algebra. Otherwise, their determination in a given experimental framework is another challenge in perspective.

\section{References}

[1] S. Audoly, G. Bellu, L. D'Angio, M. P. Saccomani, and C. Cobelli. Global identifiability of nonlinear models of biological systems. IEEE Transactions on Biomedical Engineering, 48(1):55-65, 2001.

[2] R. Brun, M. Kühni, H.R. Siegrist, W. Gujer, and P. Reichert. Practical identifiability of ASM2d parameters - systematic selection and tuning of parameter subsets. Water Research, 36(16):4113-4127, 2002.

[3] R. Brun, P. Reichert, and H. R. Künsch. Practical identifiability analysys of large environmental simulation models. Water Resources Research, 37:1015-1030, 2001.

[4] Marvin Cassman, Adam Arkin, Frank Doyle, Fumiaki Katagiri, Douglas Lauffenburger, and Cynthia Stokes. Systems Biology: International Research and Development. Springer, 2007.

[5] D. Dochain, P. A. Vanrolleghem, and M. Van Daele. Structural identifiability of biokinetic models of activated sludge respiration. Water Research, 29(11):2571-2578, 1995.

[6] M. S. Grewal and K. Glover. Identifiability of linear and nonlinear dynamical systems. IEEE Trans. on Automatic Control, 21(6):833-837, 1976.

[7] W. Hoeffding. A class of statistics with asymptotically normal distribution. The Annals of Mathematical Statistics, 19(3):293-325, 1948.

[8] T. Homma and A. Saltelli. Importance measures in global sensitivity analysis of non linear models. Realibility Engineering \& System Safety, 52(1):1-17, April 1996.

[9] H. Kitano. Foundations of systems biology. MIT press Cambridge, MA, 2001.

[10] Cleo Kontoravdi, Steven P. Asprey, Efstratios N. Pistikopoulos, and Athanasios Mantalaris. Application of global sensitivity analysis to determine goals for design of experiments: An example study on antibody-producing cell cultures. Biotechnology progress, 21(4):1128-1135, 2005.

[11] L. Ljung and T. Glad. On global identifiability for arbitrary model parametrizations. Automatica, 30(2):265-276, 1994.

[12] D. Miller and M. Frenklach. Sensitivity analysis and parameter estimation in dynamic modeling of chemical kinetics. International Journal of Chemical Kinetics, 15(7):677-696, 2004.

[13] H. Mirsky, J. Stelling, R. Gunawan, N. Bagheri, S.R. Taylor, E. Kwei, J.E. Shoemaker, and F.J. Doyle III. Automatic Control in Systems Biology. pages 1335-1360. Springer, 2009.

[14] John Peters Norton. Selection of morris trajectories for initial sensitivity analysis. In Eric Walter, editor, Proc. 15th IFAC Symposium on System Identification, volume 15, Saint-Malo, France, 2009.

[15] R. L. Peeters and B. Hanzon. Identifiability of homogeneous systems using the state isomorphism approach. Automatica, 41:513-529, 2005.

[16] H. Pohjanpalo. System identifiability based on power series expansion of the solution. Mathematical Biosciences, 41:2133, 1978.
[17] H. Rabitz and O.F. Aliş. General foundations of highdimensional model representations. Journal of Mathematical Chemistry, 25(2):197-233, 1999.

[18] D. A. Rand. Mapping global sensitivity of cellular network dynamics: sensitivity heat maps and a global summation law. J. R. Soc. Interface, 5:S59-S69, 2008.

[19] A. Raue, C. Kreutz, T. Maiwald, J. Bachmann, M. Schilling, U. Klingmüller, and J. Timmer. Structural and practical identifiability analysis of partially observed dynamical models by exploiting the profile likelihood. Bioinformatics, 25(15):1923-1929, 2009.

[20] M. P. Saccomani. Some results on parameter identification of nonlinear systems. Cardiovascular Engineering: An international journal, 4(1):95-102, 2004.

[21] M. P. Saccomani, S. Audoly, and L. D'Angio. Parameter identifiability of nonlinear systems: the role of initial conditions. Automatica, 39:619-632, 2003.

[22] A. Saltelli, M. Ratto, T. Andres, F. Campolongo, J. Cariboni, D. Gatelli, M. Saisana, and S. Tarantola. Global sensitivity analysis : The primer. Wiley, 2008.

[23] P. M. Schlosser. Experimental design for parameter estimation through sensitivity analysis. J. Toxicol. Environ. Health, 43(4):495-530, 1994.

[24] I. M. Sobol'. Global sensitivity indices for nonlinear mathematical models and their Monte-Carlo estimates. Mathematics and Computers in Simulation, 55(1-3):271-280, 2001.

[25] I. M. Sobol' and S. S. Kucherenko. Global sensitivity indices for nonlinear mathematical models. Review. Wilmott, 2005(1):56-61, 2005.

[26] IM Sobol'. Sensitivity analysis for non-linear mathematical models. Mathematical Modelling and Computational Experiment, 1(1):407-414, 1993.

[27] J. Stelling and E. D. Gilles. Robustness vs. identifiability of regulatory modules? In Proc. 2nd Intl. Conf. Systems Biology, Pasadena, USA, 2001.

[28] S. Streif, R. Findeisen, S. Waldherr, and F. Allgöwer. Systems Analysis of Biological Networks, chapter Steady state sensitivity analysis of biochemical reaction networks. A brief review and new methods. Methods in Bioengineering. Artech House, 2009.

[29] P. A. Vanrolleghem, M. Van Daele, and D. Dochain. Practical identifiability of a biokinetic model of activated sludge respiration. Water Research, 29(11):2561-1570, 1995.

[30] E. Walter and L. Pronzato. Identification of Parametric Models from experimental data. Springer, 1997.

[31] K.Z. Yao, B.M. Shaw, B. Kou, K.B. McAuley, and DW Bacon. Modeling ethylene/butene copolymerization with multi-site catalysts: Parameter estimability and experimental design. Polymer Reaction Engineering, 11(3):563-588, 2003.

[32] H. Yue, M. Brown, J. Knowles, H. Wang, D.S. Broomhead, and D.B. Kell. Insights into the behaviour of systems biology models from dynamic sensitivity and identifiability analysis: a case study of a nf- $\kappa \mathrm{b}$ signalling pathway. Mol. BioSyst., 2:640-649, 2006.

[33] D.E. Zak, G.E. Gonye, J.S. Schwaber, and F.J. Doyle. Importance of input perturbations and stochastic gene expression in the reverse engineering of genetic regulatory networks: insights from an identifiability analysis of an in silico network. Genome research, 13(11):2396, 2003.

[34] D.E. Zak, J. Stelling, and F.J. Doyle III. Sensitivity analysis of oscillatory (bio) chemical systems. Computers 85 chemical engineering, 29(3):663-673, 2005. 